\title{
Vessel Segmentation for Visualization of MRA with Blood Pool Contrast Agent
}

\author{
S. Young, V. Pekar, and J. Weese \\ 1 Philips Research Laboratories, Technical Systems Hamburg \\ Röntgenstrasse 24-26, D-22335 Hamburg, Germany \\ 2 Medical University of Lübeck, Institute for Signal Processing, \\ Seelandstrasse 1a, D-23569, Germany \\ \{stewart.young, vladimir.pekar, juergen.weese\}@philips.com
}

\begin{abstract}
We present a method for the segmentation of vessel structures in 3D magnetic resonance angiography (MRA) images with bloodpool contrast agent, allowing artery-vein separation for occluding vessel removal from MIP visualization. The method first uses a front propagation algorithm to select a path along the vessel of interest. Two controlling speed functions are considered, a multi-scale vessel filter, and an approach based on a cylinder shape model. The cylinder based method uses orientation information which is propagated with the front and iteratively updated as the surface expands. Once a vessel of interest is selected, orientation and radius parameters are used to construct a deformable model of the vessel, which is then adapted to the image borders to refine the segmentation of the selected vessel. The results of a comparison with manual segmentations are presented. The extracted centre lines are compared with those from the manual segmentations, showing a mean deviation of $2.55 \mathrm{~mm}$ for the multi-scale filter, and $1.06 \mathrm{~mm}$ for the cylinder model, compared to voxel dimensions of $0.93 \mathrm{~mm}$. The mean deviation of the final segmentation from the surface of the manual segmentation was $0.59 \mathrm{~mm}$.
\end{abstract}

\section{Introduction}

MRA images provide important information for the diagnosis of vascular diseases such as arterial stenosis and aneurysm. The visualization of the vessel pathways is crucial to allow quick and reliable assessment of any potential problems. The most common visualization method is to construct a maximum intensity projection (MIP). The recent development of MR blood-pool contrast agents which have extended intravascular halflife allows the acquisition of high resolution, high contrast 3D images of the vascular system. The longer scan times necessary to achieve higher resolution require imaging during the steady state of contrast agent diffusion. Therefore both arteries and veins are enhanced, and diagnostically important information (typically the arteries, where stenosis occurs) may be fully or partially occluded in the MIP.

Our work is aimed at semi-automated artery-vein separation. User selected seed and end points identify a vessel of interest, which is then automatically 
segmented. After segmentation, the vessel may be suppressed during generation of the MIP, revealing any previously occluded structure [10]. There are two important issues to be considered when selecting a suitable approach. Firstly, the vessel boundary should be accurately identified, since even small segmentation errors can cause residual artifacts in the MIP. Secondly, venous and arterial pathways are often close together, so methods should be able to discriminate between very closely separated structures, in order that only anatomically connected pathways are selected. A further consideration is that the segmentation method should be able to detect vessels across a range of scales, since the width of vessels can vary significantly.

Several vessel detection criteria have been proposed in the literature. A straightforward approach is to rely on image contrast, applying an intensity threshold followed by morphology analysis 8. However, non-uniform distribution of contrast agent can lead to significant intensity variations along vessels, defeating such methods. A widespread approach for vessel enhancement is to use multi-scale orientation selective filters, based on eigen-analysis of the Hessian matrix 36. Though it is asserted that these filters give their maximum response on the vessel axis, results presented here indicate this is not always observed. Krissian et al. 4 detect vessels with a boundary model based on scale and direction parameters from such a filter. An alternative boundary detection method was proposed in [12, using planar reconstructions based on orientation estimates obtained from a centre line tracking module.

As in [1, our method extracts the vessel path using a front propagation approach. However, we propose a shape-model based response as a speed function, as opposed to a purely intensity-based response which is not necessarily maximal on the vessel axis. After centre line extraction the path is combined with radius information, assuming a circular cross-section, to build a deformable model, which is then adapted to refine the segmentation. In contrast to Bulpitt and Berry [2, where the mesh structure adapts from an initial ellipsoid, our model is initialized relatively close to the object of interest, and has a fixed structure.

The next section describes the two alternative vesselness responses considered. Section 3 describes the front propagation, and the refinement method is discussed in section 4 . Validation results are presented in section 5, and conclusions are drawn in section 6.

\section{Vessel Response}

Vessels are characterized by their axial symmetry - they are extended in one direction, along their axis, but not in the directions orthogonal to this. This morphological property may be exploited to distinguish vessel voxels from other image structures.

\section{$2.1 \quad$ Multi-scale Vessel Filter}

This approach is based on analysis of local grey-value curvature, where a vessel is characterized by low derivatives along its axis, and high derivatives in orthog- 
onal directions. The Hessian matrix captures information on local second-order derivatives, and its eigenvalues reflect the degree of curvature. The eigenvector associated with the smallest eigenvalue indicates the direction of minimum curvature at a point. Therefore, assuming that the eigenvalues are ordered such that $\left|\lambda_{1}\right| \leq\left|\lambda_{2}\right| \leq\left|\lambda_{3}\right|$, and that objects of interest are brighter than the background, the response of a vesselness filter may be defined as [3]:

$$
F(\mathbf{p})=\left\{\begin{array}{l}
0, \quad \text { if } \lambda_{2}(\mathbf{p})>0 \text { or } \lambda_{3}(\mathbf{p})>0 \\
\left(1-\exp \left\{\frac{-\left|\lambda_{2}\right|^{2}}{2\left|\lambda_{3}\right|^{2} \alpha^{2}}\right\}\right) \exp \left\{\frac{-\left|\lambda_{1}\right|^{2}}{2\left|\lambda_{2} \lambda_{3}\right| \beta^{2}}\right\}\left(1-\exp \left\{-\frac{\sum_{j=1}^{3} \lambda_{j}^{2}}{2 c^{2}}\right\}\right), \text { else }
\end{array}\right.
$$

where $\alpha, \beta$ and $c$ control the sensitivity of the filter to their respective terms. The multi-scale response is defined as the maximum response over an appropriate scale range, using a scale-space representation of the image, $I(\mathbf{p}, \sigma)$, and its $\gamma$ normalized derivatives, defined as [5],

$$
\begin{aligned}
& \mathcal{I}(\mathbf{p}, \sigma)=I(\mathbf{p}) * G(\mathbf{p}, \sigma), \\
& \frac{\partial^{i}}{\partial p_{j}} \mathcal{I}(\mathbf{p}, \sigma)=\sigma^{i \gamma / 2} I(\mathbf{p}) * \frac{\partial^{i}}{\partial p_{j}} G(\mathbf{p}, \sigma)
\end{aligned}
$$

where $G(\mathbf{p}, \sigma)$ is a Gaussian kernel of width $\sigma$, and $\gamma$ is a normalization parameter. An appealing feature of this filter is that it requires no prior orientation estimate. However, the accuracy of the resulting radius estimate is typically low, since considerations of complexity, and memory requirements, restrict the number of scales which it is feasible to search. Furthermore, discrimination between nearby structures is reduced at coarser scales.

\subsection{An Adaptable Cylinder Model}

A short section of vessel may be modeled using a simple geometric cylinder representation. The cylinder is parameterized by its length, $L$, radius, $r$, and axis orientation, $\boldsymbol{a}$. Its surface, at an image position $\boldsymbol{p}$, is then specified according to

$$
\begin{aligned}
\mathbf{s}(l, \theta)=\boldsymbol{p}+l \boldsymbol{a}+r(\cos \theta \boldsymbol{b}+\sin \theta \boldsymbol{c}) \quad \text { for } \quad-\frac{L}{2} \leq l \leq \frac{L}{2}, \\
0 \leq \theta \leq 2 \pi
\end{aligned}
$$

where $\boldsymbol{b}$ and $\boldsymbol{c}$ are orthonormal vectors in the plane perpendicular to the axis, such that $\boldsymbol{a} \cdot \boldsymbol{b}=\boldsymbol{a} \cdot \boldsymbol{c}=\boldsymbol{b} \cdot \boldsymbol{c}=0$. In practice a discrete sampling of this surface, $\mathbf{s}\left(l_{i}, \theta_{j}\right)=\mathbf{s}_{i, j}$ (where $i=1, \ldots N_{l}, j=1, \ldots N_{\theta}$ ) is used. Agreement between the model and the image at $\boldsymbol{p}$ can be measured by integrating the image gradient across the surface (disregarding the ends of the cylinder). Ideally, the response at $\boldsymbol{p}$ is obtained by maximizing this measure with respect to the model parameters. However, considerations of computational complexity rule out such a direct approach.

An alternative response is defined based on model adaptation, inspired by a method for deformable mesh model adaptation [11. Assuming that an approximate direction estimate is available, a set of feature points, $\tilde{\mathbf{x}}_{i, j}$, are identified via a search along the surface normals, $\boldsymbol{n}_{j}\left(=\cos \theta_{j} \boldsymbol{b}+\sin \theta_{j} \boldsymbol{c}\right)$, according to

$$
\tilde{\mathbf{x}}_{i, j}=\mathbf{p}+l_{i} \boldsymbol{a}+\delta \boldsymbol{n}_{j} \underset{k=1 \ldots r_{\max }}{\arg \min }\left\{D k^{2} \delta^{2}-f\left(\mathbf{p}+l_{i} \boldsymbol{a}+k \delta \boldsymbol{n}_{j}\right)\right\}
$$


where $\delta$ is the spacing between points on the searched profile, $D$ is a weighting factor, $r_{\max }$ is the maximum search radius, and $f(\mathbf{x})$ is the feature value (we use the gradient in the direction of the cylinder normal). After feature point detection, cylinder parameters are updated as follows. A new axis orientation is determined as the mean orientation over all vectors between feature points at opposite ends of the cylinder for the same orientation, $\boldsymbol{d}_{j}=\tilde{\mathbf{x}}\left(l_{1}, \theta_{j}\right)-\tilde{\mathbf{x}}\left(l_{N_{l}}, \theta_{j}\right)$. The updated radius is then calculated as the mean perpendicular distance of the feature points to the updated axis.

The vesselness response should be largest on the vessel axis. Therefore, we use the RMS value of the residual distances between the feature points and the adapted cylinder surface, $\tilde{\mathbf{s}}$,

$$
R=\sqrt{\frac{1}{N_{l} N_{\theta}} \sum_{i, j}\left\|\tilde{\mathbf{x}}_{i, j}-\tilde{\mathbf{s}}_{i, j}\right\|^{2} /\left\|\tilde{\mathbf{s}}_{i, j}\right\|^{2}}
$$

where $\tilde{\mathbf{s}}_{i, j}$ are points at which the search profiles intersect the adapted model's surface. This is combined with the feature strengths, to define the speed function

$$
F(\mathbf{p})=\exp \left\{-R^{2} / \mu^{2}\right\}\left(1-\exp \left\{-\frac{1}{N_{l} N_{\theta}}\left(\sum_{i, j} f\left(\tilde{\mathbf{x}}_{i, j}\right)\right) / \nu\right\}\right)
$$

where the constants $\mu$ and $\nu$ serve to normalise the exponential terms around expected values for the RMS fractional deviation and mean feature value respectively, and to control the sensitivity of surface evolution to the respective terms. In our experiments, these values were set to $\mu=0.17$ and $\nu=30.0$.

\section{Centre Line Extraction via Front Propagation}

A front propagation approach is well suited to selecting vessel structures, where a simple local structure is repeated to form a complex pattern at larger scales. The method [7] is initialized by selecting a seed point inside the object of interest. The initial state of a time field, $T(\mathbf{x})$, is defined as zero at the ('selected') seed point, and infinity at all other ('un-selected') locations. Subsequently, the propagation of the front proceeds by iterating the following operations:

1. Voxels in the un-selected region which border the selected region are labeled as border, and their time values are updated according to the governing equation:

$$
|\nabla T| F=1
$$

2. The voxel in the border with the lowest time value is moved to the selected region.

The use of the multi-scale Hessian filter as the speed function is straightforward. In the case of the cylinder model based response, initial orientation parameters 
are estimated at the seed point via an exhaustive search, then propagated as voxels are moved into the border set, and updated whenever the time is computed.

If an end-point is selected before propagation, the process is terminated when this point is reached. Otherwise, a fixed number of iterations are used, and an end point is then interactively obtained from the selected region. A path may be constructed between the seed and end points, by following the minimum time connections in the time field.

\section{Deformable Model Refinement}

The centre line extracted via front propagation is used to reconstruct the vessel volume. If the cylinder-based speed function is used, orientation and radius estimates are available directly, otherwise cylinder models may be oriented along the path and adapted to retrieve these estimates. However, a vessel's cross sectional profile often deviates from circular. Visualization applications require accurate detection of the vessel wall, in order to avoid residual regions appearing in the MIP. Therefore, we construct a deformable model [9] using the centre line and radius estimates, which can be adapted to refine the segmentation.

The vessel is represented using a triangulated mesh, which is adapted according to image features while also imposing shape based constraints on the deformation. The chosen method [1] maintains the underlying triangle structure, and uses the initialising configuration as a guiding shape model to avoid excessive deformation. Adaptation is an iterative procedure consisting of a surface detection step, similar to the feature detection described in equation (4), followed by minimization of an energy function. The energy is composed of an external, image-related energy, and an internal, shape-related term:

$$
E(\mathbf{x}):=\alpha E_{\text {int }}(\mathbf{x})+E_{\text {ext }}(\mathbf{x})
$$

where $\alpha$ weights the relative influence of each term. The external energy attracts the mesh towards the surface points. The internal energy is defined with respect to changes of the difference vectors between neighbouring mesh vertices, penalizing large deviations from the initial shape. Energy minimization uses the conjugate gradients method.

\section{Results}

The approach was tested with blood-pool MR images, and a validation performed via comparison with manual segmentations. Reference segmentations of the major venous and arterial pathways were available for two images. The images were re-sampled to obtain isotropic data from original image dimensions of $512 \times 512 \times 60$ voxels of size $0.9 \times 0.9 \times 1.8 \mathrm{~mm}$.

Centre lines estimates extracted for both speed functions were compared to vessel axes obtained from the manual segmentations. Two scales were used for 


\begin{tabular}{|c|c|c||c|c||c|c|}
\hline \multirow{2}{*}{ Data set } & \multicolumn{2}{|c|}{ Mean deviation (mm) } & \multicolumn{2}{c|}{ Max. deviation (mm) } & \multicolumn{2}{c|}{ Path length (mm) } \\
\cline { 2 - 7 } & CYL & MULTI & CYL & MULTI & CYL & MULTI \\
\hline 1 - right vein & 1.110 & 2.836 & 3.085 & 10.230 & 486.4 & 479.0 \\
\hline left vein & 0.910 & 2.458 & 2.941 & 7.143 & 501.3 & 475.2 \\
\hline 2 - right vein & 0.900 & 2.582 & 3.222 & 6.307 & 535.7 & 537.5 \\
\hline left vein & 1.330 & 2.342 & 8.823 & 9.206 & 458.5 & 439.9 \\
\hline
\end{tabular}

Table 1. Comparison of mean and maximum deviations of steepest descent path with respect to centre-line of manually segmented data

the multi-scale filter response, the original image resolution and $\sigma=2$, and the parameters were set to $\alpha=\beta=0.5, c=5$, while for the cylinder model approach, the parameters used were $L=4, D=0.1$. Reference paths were extracted using front propagation, with a speed function defined using the distance map of the manual segmentation, such that propagation was fastest at voxels furthest from the background. Then the minimal time path between the seed and end point was computed to define the reference centre line. For each point of the automatically determined centre line, the minimum distance to the reference centre line was computed. Comparison results for the two speed functions are given in table 1 . The mean error for the cylinder fitting method is $1.06 \mathrm{~mm}$, compared to $2.55 \mathrm{~mm}$ for the multi-scale vessel filter.

The paths extracted using the cylinder-based speed function were used to construct deformable models. The final segmentations after model adaptation were compared to the manual segmentations in two ways: using the overlap between the volumes, and the mean Euclidean distance between the surfaces of the two volumes. Since each manual segmentation also included several other vessel sections connected to the vein of interest at bifurcations, the regions near bifurcations were masked from the following results, in order to avoid ambiguity in defining voxels belonging to the vessel of interest. Comparison results are shown in table 2 Rather than considering the total proportion of falsely selected background voxels, we compute the fraction of artery falsely included since this is the important information regarding the appearance of the MIP. There are two important considerations when assessing these results. Firstly, there is a degree

\begin{tabular}{|c|c|c|c|c|}
\hline Data set & $\begin{array}{c}\text { Vein volume } \\
\text { (voxels) }\end{array}$ & $\begin{array}{c}\text { Vein segmented } \\
\text { (\% of total) }\end{array}$ & $\begin{array}{c}\text { Artery included } \\
\text { (\% wrt vein volume) }\end{array}$ & $\begin{array}{c}\text { Mean Deviation } \\
\text { (mm) }\end{array}$ \\
\hline 1 - right vein & 40783 & 87.86 & 1.10 & 0.586 \\
\hline left vein & 42510 & 88.94 & 0.68 & 0.516 \\
\hline 2 - right vein & 52593 & 85.49 & 0.47 & 0.632 \\
\hline left vein & 40845 & 87.24 & 0.59 & 0.622 \\
\hline
\end{tabular}

Table 2. Comparison of the overlap, and Euclidean distances, between the final and the manual segmentations. 

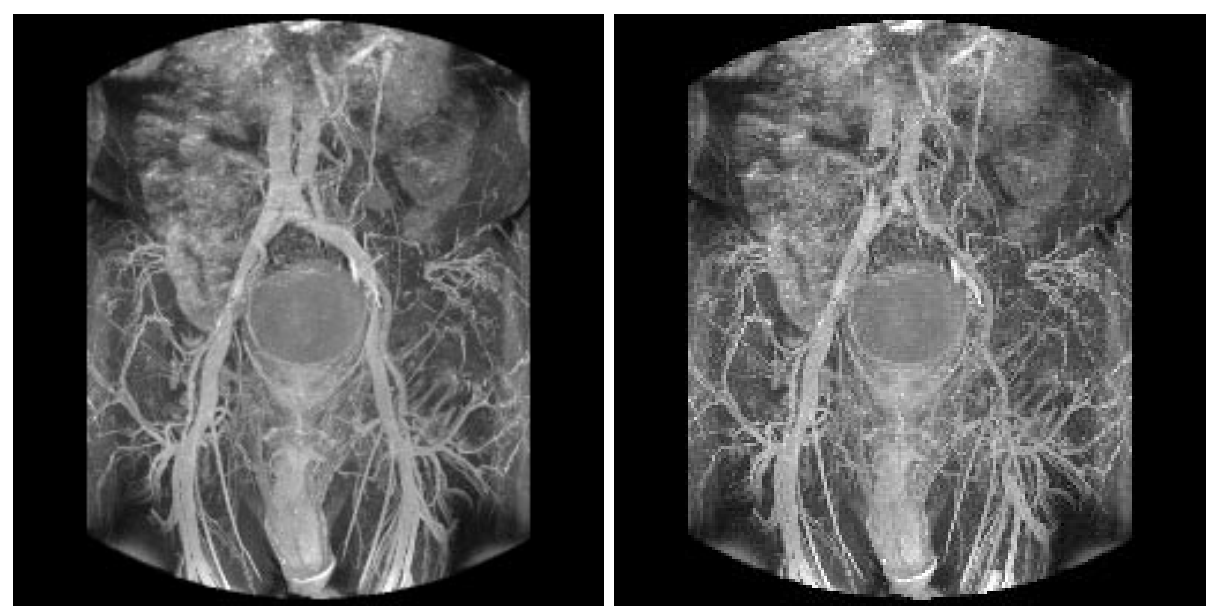

Fig. 1. MIPs before (left) and after (right) suppression of the right hand vein selected using our method, with the cylinder-model based speed function.

of subjectivity during hand segmentation, regarding the placement of the vessel border. Secondly, vessel structures have a large surface area to volume ratio, and so small errors in the radius estimate can lead to a significant error in the segmented volume. For example, the veins considered here have an average radius of approximately $5 \mathrm{~mm}$, and an error of $0.5 \mathrm{~mm}$ in the average radius estimate would result in a $20 \%$ error in the selected volume. The mean distance between the automatic and reference surface, which is less than the voxel dimensions, shows that the final segmentation accurately identifies the vessel boundaries. Figure 1 shows two MIPs, before and after suppression of right hand vein, where the cylinder model based speed function was used. Apart from a small region of unselected vein seen just below the artery bifurcation, where the deformable model failed to identify the vein border, the artery pathway is clearly seen after suppression.

\section{Conclusions}

A method for vessel selection has been described which allows individual vessels to be suppressed from MIP visualizations. First, a centre line is determined using a front propagation method guided by a cylinder model based response, where model parameters are propagated with the front, and then updated. The resulting centre line is combined with radius and orientation information to construct a deformable model which is adapted to refine the segmentation. The cylinder-based vessel detection criterion improves discrimination between the vessel of interest and other nearby structures, compared to a multi-scale filtering criterion. A validation of the method shows that the centre line extracted has 
a mean deviation of $1.06 \mathrm{~mm}$, and that the final segmentation has a mean error of $0.59 \mathrm{~mm}$. While further improvements are necessary, for example for accurate border selection in regions near bifurcations, we have demonstrated the feasibility of artery-vein separation.

\section{Acknowledgments}

The authors thank Dr. Toombs and Dr. Flamm from St. Luke's Episcopal Hospital, Houston, USA for providing MRI blood pool contrast images. We would also like to thank Arianne van Muiswinkel and Jan de Becker from Philips Medical Systems, Best, Netherlands for their support.

\section{References}

1. B. B. Avants and J. P. Williams. An adaptive minimal path generation technique for vessel tracking in CTA/CE-MRA volume images. In MICCAI'00, pages 707716, Pittsburgh, PA, 2000.

2. A. Bulpitt and E. Berry. An automatic 3D deformable model for segmentation of branching structures compared with interactive region growing. In Med. Image Understanding and Analysis, pages 25-28, Leeds ,UK, 1998.

3. A. Frangi, W. Niessen, K. Vincken, and M. Viergever. Multiscale vessel enhancement filtering. In MICCAI'98, pages 130-137, Cambridge, MA, 1998.

4. K. Krissian, G. Malandain, N. Ayache, R. Vaillant, and Y. Trousset. Model based detection of tubular structures in 3D images. Technical Report TR-3736, INRIA, July 1999.

5. T. Lindeberg. Edge detection and ridge detection with automatic scale selection. International Journal of Computer Vision, 30(2), 1998.

6. C. Lorenz, I.-C. Carlsen, T. Buzug, C. Fassnacht, and J. Weese. Multi-scale line segmentation with automatic estimation of width, contrast and tangential direction in 2D and 3D medical images. In Proc. of CVRMed-MRCAS '97, pages 233-242, Grenoble, France, 1997.

7. R. Malladi and J. A. Sethian. A real-time algorithm for medical shape recovery. In Proc. 8th Int. Conf. on Computer Vision, pages 304-310, Bombay, India, 1998.

8. Y. Masutani, T. Schiemann, and K.-H. Höhne. Vascular shape segmentation and structure extraction using a shape-based region-growing model. In MICCAI'98, pages 1242-1249, Cambridge, MA, 1998.

9. T. McInerney and D. Terzopoulos. Deformable models in medical image analysis: A survey. Medical Image Analysis, 1(2):91-108, 1996.

10. W. Niessen, A. Montauban van Swijndregt, B. Elsman, O. Wink, M. Viergever, and W. Mali. Improved arterial visualization in blood pool agent MRA of the peripheral vasculature. In $C A R S^{\prime} 99$, pages 119-123, 1999.

11. J. Weese, M. R. Kaus, C. Lorenz, S. Lobregt, R. Truyen, and V. Pekar. Shape constrained deformable models for 3-D medical image segmentation. Accepted for IPMI 2001, June 2001.

12. O. Wink, W. J. Niessen, and M. A. Viergever. Fast delineation and visualization of vessels in 3D angiographic images. IEEE Trans. Medical Imaging, 19(4):337-346, 2000. 